\title{
A Visible Light Localization Aided Optical Wireless System
}

\author{
Giulio Cossu, Marco Presi, Raffaele Corsini, Pallab Choudhury, Amir Masood Khalid and Ernesto Ciaramella \\ Scuola Superiore Sant'Anna University - TeCIP \\ Via G. Moruzzi, 1, 56124 - Pisa (ITALY) \\ Email: giulio.cossu@sssup.it
}

\begin{abstract}
We report about a Line of Sight Optical Wireless Communication (LoS OWC) system aided by a simplified Visible Light localization algorithm for tracking purposes. We show experimentally that by combining the LoS OWC system with the localization algorithm it is possible to provide a gross datarate exceeding $300 \mathrm{Mb} / \mathrm{s}$ over a $90^{\circ}$ illumination angle by using a RGB visible-light $L E D$ at $90 \mathrm{~cm}$ distance.

Index Terms-Visible Light Communications, LED, Adaptive OFDM, Localization
\end{abstract}

\section{INTRODUCTION}

Optical Wireless Communication (OWC) systems have been recently investigated for different applications, ranging from sensing [1] to data-transmission [2]. Among OWC systems, those based on Visible Light are of particular interest for indoor environments since they can combine illumination and communication functionalities. A great effort has been put to demonstrate different applications based on white LEDs (either phosphor-based and RGB).

For communication purposes, Visible Light Communication (VLC) record has been demonstrated at data rates up to $803 \mathrm{Mb} / \mathrm{s}$ by combining advanced Digital Signal Processing and Wavelength Division Multiplexing in RGB LEDs [3]. Yet, this requires a line-of-sight (LoS) system with a focalization lens. VLC systems based on white LEDs and fluorescent lights have been also demonstrated to be effective for localization purposes, as an aid for visually impaired people or to provide localization information in indoor environment, where GPS is not available [4], [5], [6]. These systems can provide up to sub-centimeter accuracy [7].

Recently, an interesting proposal suggested to exploit localization techniques based on common radio infrastructures (eg. WiFi) as an aid to provide tracking information in widearea LoS optical wireless systems [8]. In this case, thanks to a transceiver design that relaxes the fiber-to-fiber alignment [9], a rough localization information (about half-a-meter) is enough, and the radio-based protocol can easily fulfill these requirements [10]. However such a resolution doesn't meet the requirements of high bit-rate VLC systems. Indeed the very limited modulation bandwidth of visible light LEDs (usually less then $20 \mathrm{MHz}$ [11], [12]) and the relatively low detector responsivity in the visible region, make this class of systems very sensitive to Signal to Noise Ratio (SNR) degradation: this poses more stringent requirements on the transmitter-receiver alignment.

Those requirements are even more strict when spectrally efficient modulation formats, such as adaptive Orthogonal Frequency Division Multiplexing (OFDM), are implemented in to increase the net data-rate [13]. In this case to meet the SNR requirements, the receiver needs a focalization lens which drastically decreases the alignment tolerance angle down to few degrees. Fortunately, the relatively low modulation bandwidth of visible light LEDs allow to use large area photodiodes, thus avoiding further SNR and alignment tolerance constraints.

We therefore propose to use a localization algorithm based on visible light. We demonstrate experimentally that the localization accuracy obtained by our solution is fully compatible with the angle tolerance required by our focalized LoS VLC system based on adaptive OFDM. A proof of principle of this technique is demonstrated in a 1-D system. By means of this approach we achieve a localization error that is low enough to guarantee a data-rate between 300 and $410 \mathrm{Mb} / \mathrm{s}$ using only one transmitting RGB LED, covering an illumination angle of $90^{\circ}$ and at $90 \mathrm{~cm}$ link length.

\section{OPERATING PRINCIPLE}

We consider a room where LEDs installed on the ceiling provide simultaneously for illumination, localization and data transmission. A minimum of three LEDs is required for the localization, while at least one LED lamp is required for the data transmission. A general scheme of the proposed method is reported in Fig. 1. In this case a system with 4 auxiliary transmitters is shown. Data Tx is the LED used for the data transmission, Data $R x$ is the data receiver with focalization lens, $T x_{i},(i=1, \ldots, 4)$ and $R x$ are respectively the auxiliary transmitters and receiver used for localization purpose. Data $T x$ and $R x$ are as close as possible. As said, the lens in $R x$ increases the SNR and improves the system capacity; on the other hand, it reduces drastically the acceptance angle for the incoming signal so it must be carefully aligned with the Data Tx (LoS). In order to obtain the required LoS between Data $T x$ and Data Rx, the two coordinates $\left(d_{x}, d_{y}\right)$ must be known with an error well below the tolerance of the system. The $R x$ does not use any focalization system, in order to have a wide acceptance angle. 


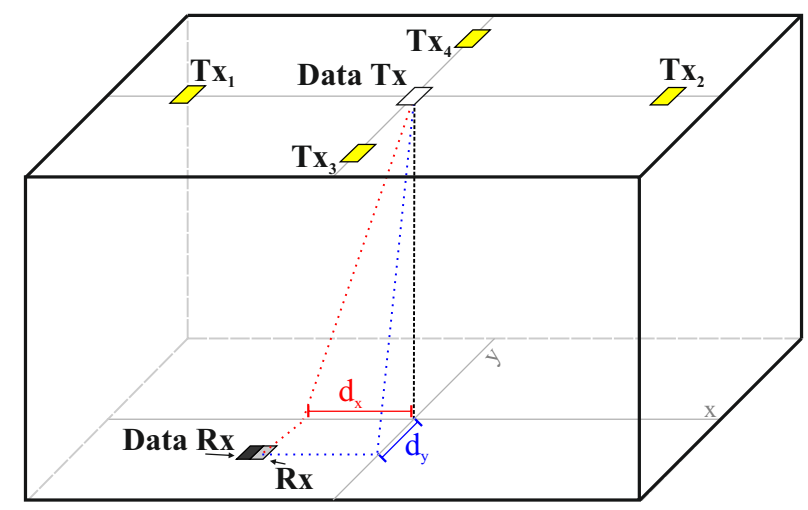

Figure 1. Scheme of the proposed 2-D localization method with 4 auxiliary transmitters.

Let us explain in detail our technique considering a 1-D system. This is reported in Fig. 2 where $L_{i}(i=1,2)$ are the distances between $T x_{i}(i=1,2)$ and $R x ; h$ is the distance between the planes of the transmitters and the receiver and $\Omega$ is the angle at which the Data Rx should point in order to be in LoS with Data Tx. The receivers Rx and Data Rx are placed at coordinate $d$ that should be determined by the proposed localization algorithm.

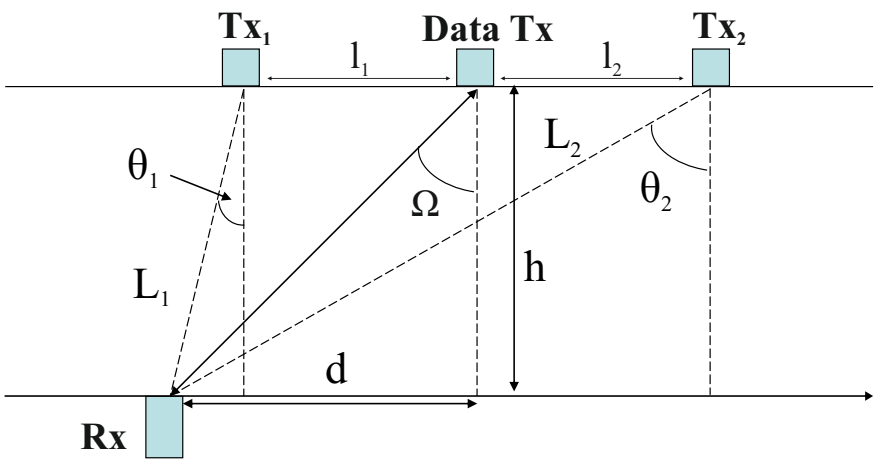

Figure 2. Description of 1-D localization algorithm.

The LEDs used for the localization should also transmit a topology information, in order to provide the position of the $T x_{i}$. For localization purposes, each reference LED transmits a unique identifier. In our approach the unique ID is represented by a single tone. Each tone is located at frequencies lower than $700 \mathrm{kHz}$, in a frequency region where there is no overlap with the high data-rate signal. The data transmission is achieved by using an adaptive OFDM signal, also known as Discrete Multi-Tone (DMT), over a bandwidth of $90 \mathrm{MHz}$.

In order to establish the VLC link, the following steps must be followed. The receiver first detects the number of reference LEDs, by counting the number of modulated tones. Then the receiver computes its position within the room by measuring the relative power emitted by each localization LED using the propagation model described in in the following, and correlating it with the location map of the localization LED, i.e. the coordinates $l_{i}$ (see Fig. 2). To this aim we recall that, in the case of a single source, the power at the receiver is given by the expression:

$$
P(\theta, \psi)=P(0,0) \frac{h^{2} \cos ^{m}(\theta) \cos ^{M}(\psi) T_{s}(\psi) g(\psi)}{L^{2}}
$$

where $\theta$ is the angle of irradiance, $\psi$ is the angle of incidence at the $R x, T_{s}(\psi)$ is the gain of an optical filter and $g(\psi)$ is the gain of an optical concentrator placed in front of the detector [14]. $P(\theta=0, \psi=0)$ is the received power at distance $h$. In this paper, we assume that both LED chip and detector have a Lambertian radiation pattern with different order, $m$ and $M$ respectively; moreover $T_{s}(\psi)=g(\psi)=1$ because no filter and lens are being used at the $R x$.

When both $T x_{i}$ and $R x$ are perpendicular to the floor we have $\psi=\theta$. In order to account for multiple reflections, we consider a path-loss exponent correction factor $\gamma$ [14]. Then the Eq. 1 becomes, for the two $T x_{i}$ :

$$
P_{i}\left(\theta_{i}\right)=P_{i}(0) \frac{h^{\gamma} \cos ^{m}\left(\theta_{i}\right) \cos ^{M}\left(\theta_{i}\right)}{L_{i}^{\gamma}} i=1,2
$$

We used a value of $\gamma=4$ as it fits well the typical range considered for indoor scenarios [15]. By using the relation $L_{i}=\frac{h}{\cos \left(\theta_{i}\right)}$, Eq. 2 can be rewritten as:

$$
P_{i}\left(\theta_{i}\right)=P_{i}(0) \cos ^{m+M+\gamma}\left(\theta_{i}\right) \quad i=1,2
$$

Using two auxiliary transmitters, as in Fig 2, we can remove the ambiguity in respect to the symmetry of the sources. Eq. 3 allows thus to individuate univocally the angles $\theta_{i}$, provided that we have $\theta_{i}<\frac{\pi}{2}$. Measuring $P_{i}\left(\theta_{i}\right)$ and knowing $P_{i}(0)$ and the parameters $m$ and $M$ it is possible to obtain $\theta_{i}$ inverting Eq. 3 . Once the couple $\theta_{i}$ has been determined, we obtain both the displacement position $d$ and the angle $\Omega$ using the known values of $l_{i}$ and a simple trigonometric relationship. For a 2-D system, the procedure must be modified, although it retains the same working principle of the 1-D case. In Fig. 3 we report a top-view of the system: the Data Tx (white box) is placed at coordinates $(0,0)$. The $T x_{i}$ are placed along $X$ and $Y$ axes. The $R x$ is indicated by a gray box and placed at coordinates $\left(d_{x}, d_{y}\right)$. At these coordinates, each $T x_{i}$ delivers a fixed optical power as defined in Eq. 3: however, this information defines a circle of constant power for each one of the $T x_{i}$. The coordinates $\left(d_{x}, d_{y}\right)$ can be then evaluated by calculating the intersection point of those circles. Once $\left(d_{x}, d_{y}\right)$ are known, the data receiver can change its pointing angle toward the transmitter, establishing a LoS link. A detailed derivation of the 2-D localization algorithm is currently under development.

Of course, the localization accuracy increases by increasing the number of reference LEDs. A minimum of 3 reference LEDs is required for 2-D localization. In order to ensure $\mathrm{LoS}$ operation, the receiver should be able to change its orientation. This requires some adaptive optics, e.g. a steering mirror, or an electrical actuator able to rotate the lens-receiver pair over two axis.

\section{EXPERIMENT}

The proposed method has been experimentally investigated as described in this section. Due to the lack of a large number 


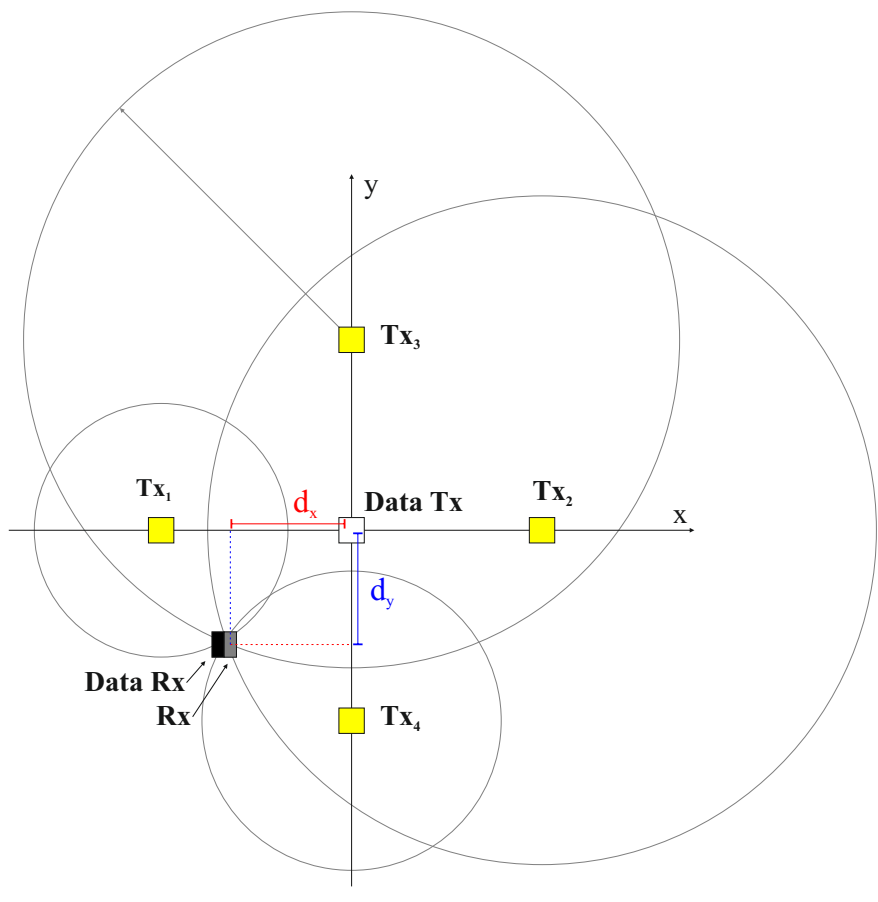

Figure 3. Top View of the 2-D system: by using the power information from each auxiliary LEDs (yellow), it is possible to find the coordinates of the $R x$ (gray-box) in respect to the Data Tx (white box) placed at coordinates $(0,0)$.

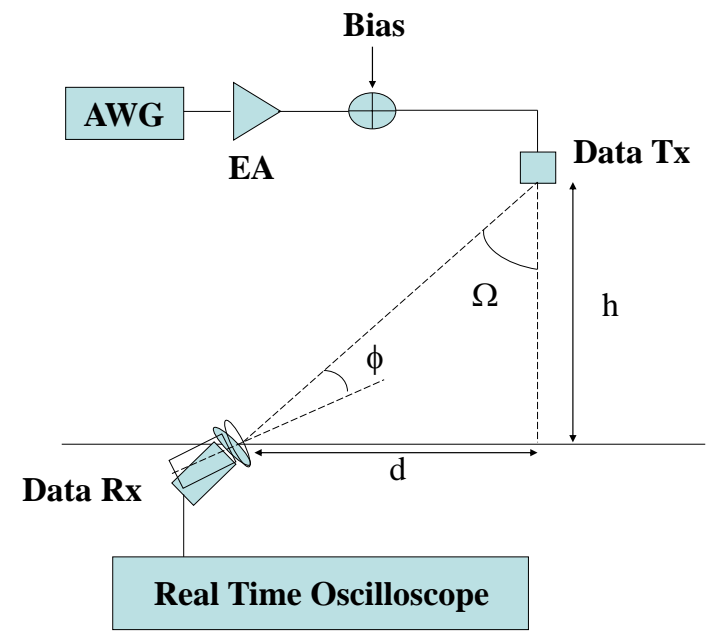

Figure 4. Experimental Setup used to determine the angular tolerance to LoS mismatch of the VLC link. AWG: Arbitrary Waveform Generator; EA: Electrical Amplifier.

of LEDs, we restrict our proof-of-concept demonstration to a 1-Dlocalization and a manually adapted receiver.

As a preliminary step, we measured the performances of the data system, as the gross capacity vs. $d$ and vs. angle $\Omega$ at a $2 \times 10^{-3}$ Bit Error Ratio (BER) as defined in Fig. 2. To this aim, we used the setup presented in Fig. 4. This measurement allowed to evaluate the receiver (Data $R x$ ) tolerance to the deviation of the pointing angle $\phi$ in order to provide the specification to the proposed localization method. The transmitter (Data $T x$ ) was a commercially available white

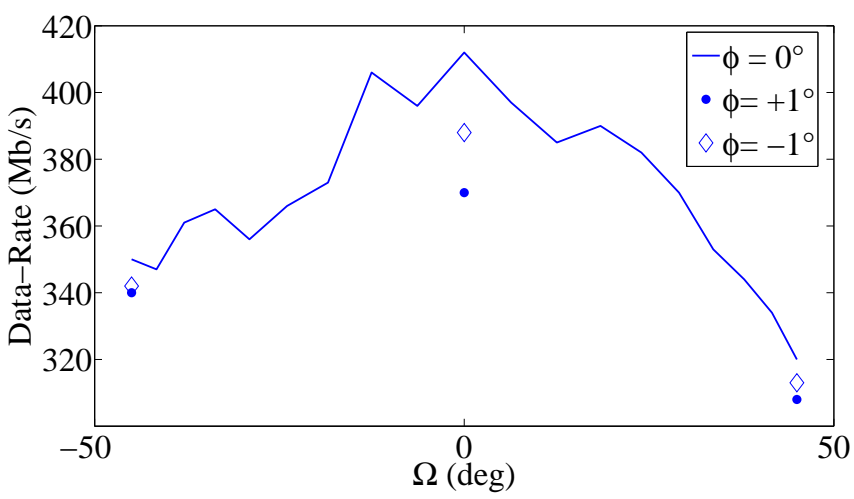

Figure 5. Experimental values of link data-rate vs displacement angle $\Omega$. For optimal pointing angle $\left(\phi=0^{\circ}\right.$, continous line). and at $\phi \pm 1^{\circ}$ (dots).

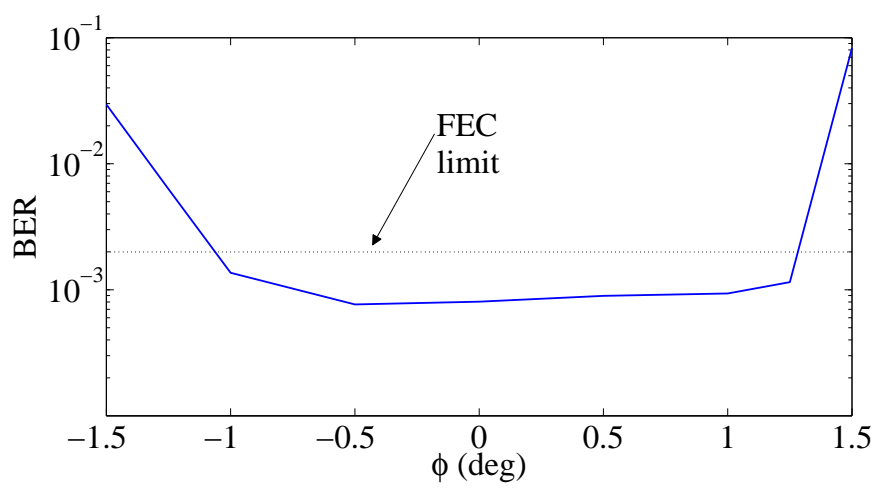

Figure 6. BER as a function of the error on the pointing angle $\phi$.

composite RGB LED luminary (generating a luminous flux of $105 \mathrm{~lm}$ at typical driving currents of $350 \mathrm{~mA}$, with a radiation angle of $120^{\circ}, 12 \mathrm{MHz}$ modulation bandwidth), consisting of three chips radiating in the wavelength regions of $625 \mathrm{~nm}$ (red), $525 \mathrm{~nm}$ (green) and $460 \mathrm{~nm}$ (blue). In order to drive all $3 \mathrm{LED}$ chips simultaneously, they have been connected in series. The DMT signal from the Arbitrary Waveform Generator (AWG) output was then used to modulate the 3 LED chips under test. Before the modulation of optical sources, the AWG output signal was amplified (Mini Circuits ZHL 32A) and via a Bias-T combined with the bias current (190 mA). The bias value was chosen in order to optimize the link performance.

After transmission over the visible-light channel a commercially available silicon APD (Hamamatsu model C5331-11, $1 \mathrm{~mm}$ active area diameter, $100 \mathrm{MHz}$ bandwidth) integrated with a trans-impedance amplifier was used for detection of the optical signal. In addition we used a glass lens $(25 \mathrm{~mm}$ focal length and $25 \mathrm{~mm}$ diameter) in front of the APD in order to increase the SNR of the received signal. The electrical signal was acquired by a real time sampling oscilloscope for off-line post processing (including equalization and BER evaluation).

The DMT signal consisted of $N=128$ sub-carriers within a bandwidth $B=90 \mathrm{MHz}(0.703 \mathrm{MHz}$ sub-carrier spacing). Bit and power loading was applied on $N-1$ sub-carriers (excluding the DC component).

In all the following measurements, the distance $h$ was fixed 
at $90 \mathrm{~cm}$. At this distance, the brightness level was measured by a light-meter placed in front of the receiver and was found to be $30 \mathrm{~lx}$ (background light subtracted) for $d=0$. This conditions is well below the value recommended by the standard for the working environments (500 lux) [16].

The maximum gross capacity (bit rate) at a BER of $2 \times 10^{-3}$ (FEC limit), was measured as a function of the position $d$ (i.e. angle $\Omega$ ) for a pointing angle $\phi=0^{\circ}$ (i.e. exact pointing toward the transmitter). The same data-rate measurement vs. pointing angle $\phi$ was carried-out at three representative displacements $d$ : the zero-displacement position $\left(\Omega=0^{\circ}\right)$ and the two edge positions $\left(\Omega= \pm 45^{\circ}, d= \pm 90 \mathrm{~cm}\right)$. The results are summarized in Fig. 5 where the continuous line shows the data-rate obtained for a BER of $2 \times 10^{-3}$ as a function of the displacement angle $\Omega$, with $\phi=0^{\circ}$ (best pointing condition). The diamonds and circles indicate the data-rate at $\Omega=0, \pm 45^{\circ}$, for $\phi=0, \pm 1^{\circ}$ respectively; we noted that when the pointing error exceeds $\pm 1^{\circ}$ the received signal drastically drops and no further measurement can be performed. However, as we see, a minimum capacity of $300 \mathrm{Mb} / \mathrm{s}$ was always guaranteed across the whole displacement range, with a maximum value of $410 \mathrm{Mb} / \mathrm{s}$. The DMT adaptation algorithm was run at each $\Omega$ value, thus the bit and power loading configuration was different for each point.

In Fig 6 we report a detailed analysis of the $R x$ tolerance to the pointing angle $\phi$. In this case the data rate was fixed, the $R x$ was at $\Omega=0^{\circ}$. In Fig. 6 we report the measured BER vs $\phi$; from this we see that for $\phi= \pm 1^{\circ}$ the BER variation is below half a decade. Further increasing the error of the pointing angle results into a drastic performance decrease. We thus conclude that the tolerance of the pointing angle is around $\pm 1^{\circ}$. The asymmetry in the curve is due to a non perfect alignment between the lens and APD receiver.

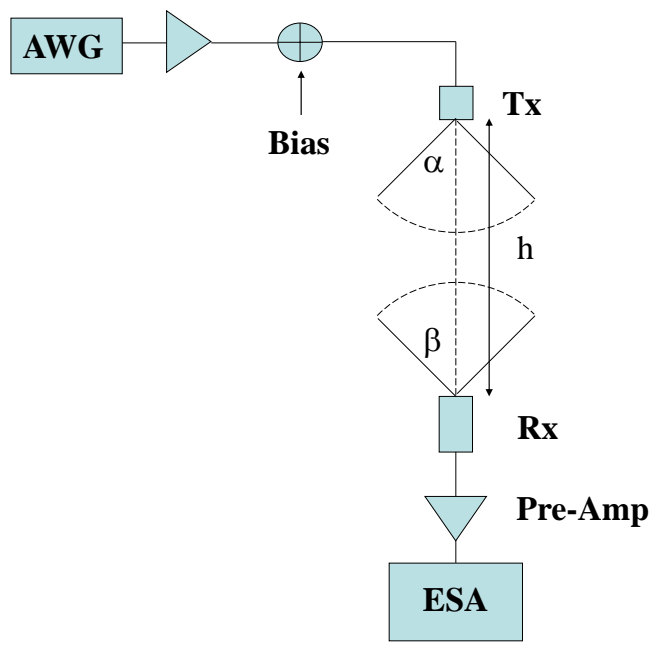

Figure 7. Setup for the characterization of the radiation lobe and the acceptance angle of the auxiliary transmitter and receiver.

We then measured the radiation pattern of the auxiliary transmitters and the acceptance angle of the auxiliary receiver (indicated in the following as $T x_{i}, i=1,2$ and $R x$ respec- tively). The auxiliary transmitter was a phosphorescent white LED (generating a luminous flux of $220 \mathrm{~lm}$ at driving current of $700 \mathrm{~mA}$ ). The LED was incorporated in a polymeric lens that narrowed its radiation pattern. The auxiliary receiver was a $\mathrm{Si}$ photodiode (with average $0.3 \mathrm{~A} / \mathrm{W}$ responsivity across the LED emission spectrum) followed by a variable gain high speed amplifier. For this measurements both the LED and the photodiode were mounted on steering plates and placed each in front of the other (see Fig. 7 that illustrates the setup). In this way, both the LED and the photodiode could be rotated independently around their axes. We define $\alpha$ and $\beta$ as the LED and photodiode rotation angles. The LED radiation angle was measured by rotating it around its axis, i.e. varying $\alpha$, while keeping the photodiode at $\beta=0^{\circ}$; similarly, the receiver acceptance angle was measured by rotating the PD respect by the angle $\beta$ at $\alpha=0^{\circ}$.

The signal from a function generator at a frequency outside the DMT signal spectrum was used to drive the LED. The DC driving current was $300 \mathrm{~mA}$ corresponding to the best LED working point. The signal was acquired by means of an electrical spectrum analyzer (ESA) and the power of the modulating carrier was measured. The collected data were fitted by a $\cos ^{k}(\cdot)$ function (we used the Lambertian approximation for both the LED and PD [14]). We found that this is a good approximation in the range of $\pm 55^{\circ}$ (see Fig. 8). The measured Lambertian emission order $m$ for both LEDs is found to be 2.99 , while an order $M=2$ fits well the $\mathrm{Si}$ photodetector acceptance angle. The effects of the variations of radiation pattern for different LED models will be the subject of future investigations.
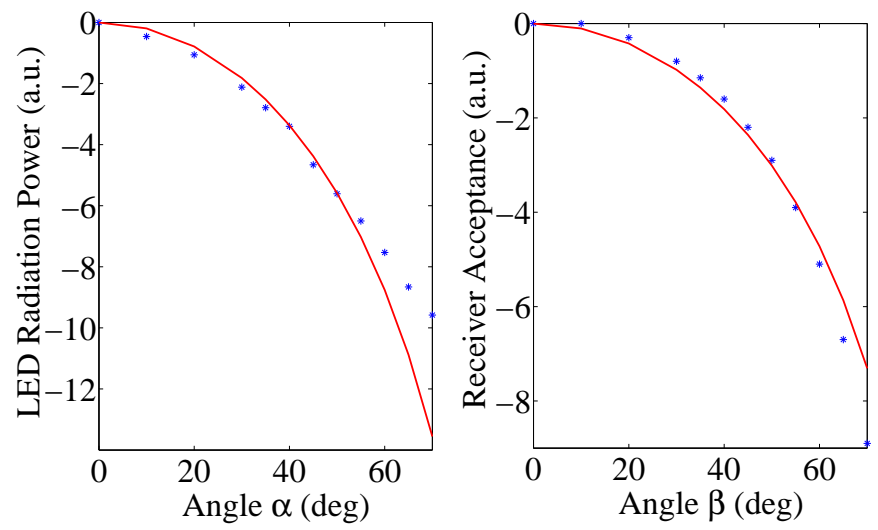

Figure 8. Experimental measurement (stars) of the Lambertian radiation pattern of the reference LEDs (left) and angular acceptance of the receiver (right). Both curves are fitted with the a Lambertian function (solid line).

Finally the setup described in Fig. 9, was realized in order to evaluate the effectiveness of the proposed pointing system. The two $T x_{i}$ were positioned symmetrically, at a distance of $45 \mathrm{~cm}$ from the Data $T x$ used for high-capacity transmission. The receiver $R x$ was set at a distance $h=90 \mathrm{~cm}$ from the line of the sources. The receiver was mounted on a guide and can be moved at arbitrary $x$ positions along $X$ axis. For each $x$ position, it is possible to define the following 


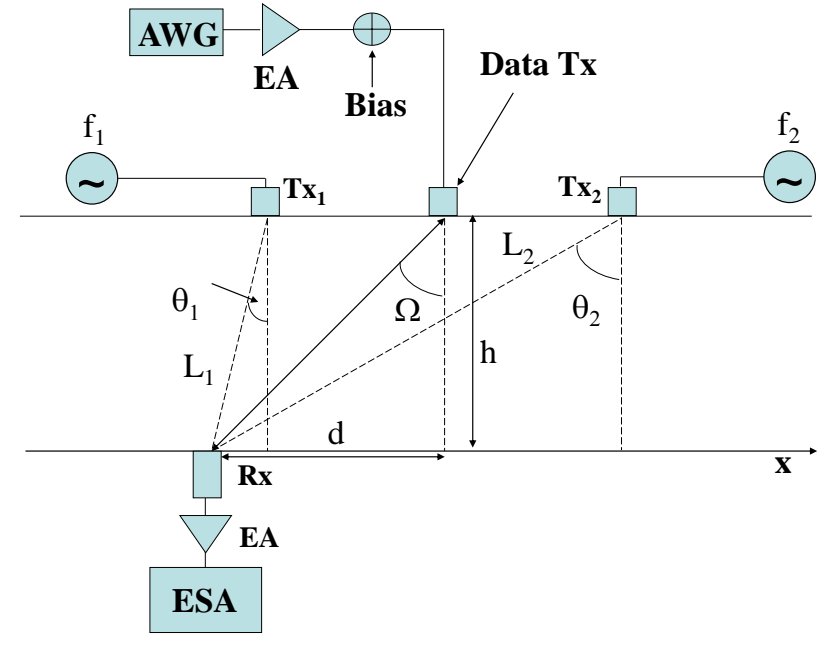

Figure 9. Complete Setup of the localization aided high-capacity VLC link. Si PD: Si Photodiode; ESA: Electrical Spectrum Analyzer.

parameters: the angle $\Omega$, i.e the angle formed by the RGB LED and the receiver; the distances $L_{1}$ and $L_{2}$ between the auxiliary receiver and the two auxiliary transmitters; the angles $\theta_{1}$ and $\theta_{2}$, i.e. the angles formed between the auxiliary receiver and the auxiliary transmitters $\left(T x_{1}\right.$ and $\left.T x_{2}\right)$. The two $T x_{i}$ were modulated by a single frequency tone at $f_{1}=500$ and $f_{2}=520 \mathrm{kHz}$ respectively. A first calibration was required, so the $R x$ was positioned in front of the two $T x_{i}$ and the power $P_{i}\left(\theta_{i}=0\right), i=1,2$, was measured. By moving the $R x$ along the $X$ axis and measuring $P_{i}\left(\theta_{i}\right)$, the two angles $\theta_{i}$ can be obtained inverting numerically the two equations 3 .

The indices $M$ and $m$ were determined by the previous measurement. Once the couple $\theta_{i}$ has been determined through Eq. 3, it is possible to obtain both the displacement position $d$ and the angle $\Omega$ by using a simple trigonometric relationship.

We define $\Delta \Omega=\left|\Omega-\Omega_{m}\right|$ as the difference between the angle $\Omega$ evaluated by the proposed method and the angle $\Omega_{m}$ geometrically measured on the setup. In Fig. 10, we report an experimental measure of $\Delta \Omega$ as a function of $\Omega$. As can be seen the corresponding pointing error was always below $1^{\circ}$ as required by the data link specification (see Fig. 10). We note that the estimation error is maximum when the receiver $R x$ is in front one of the $T x_{i}$. As can be seen from Eq. 3, in these positions $\theta=0$ so that the transmission function $\cos ^{m+M+\gamma}(\theta)$ is maximum and its derivative against the displacement is close to zero. This results clearly in a greater position estimation error. As can be seen from Fig. 10, the estimation error is not exactly symmetrical. This can be due to several reasons. For example the two reference LEDs can have a slightly different radiation pattern. Notwithstanding, the overall error is within the tolerances as reported in Fig. 4.

\section{CONCLusions}

We presented the experimental proof-of-principle of a VLC system based on RGB LEDs capable of providing a data-rate between 300 and $400 \mathrm{Mb} / \mathrm{s}$ within an illumination angle of $90^{\circ}$

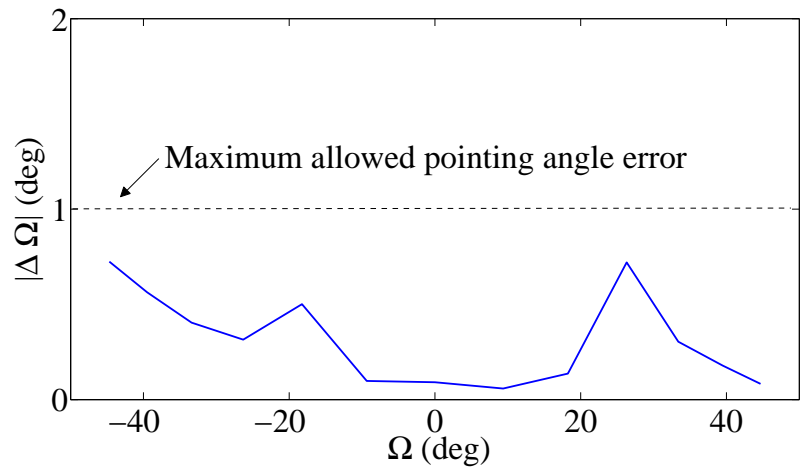

Figure 10. Experimental measurement of the pointing angle estimation error.

at a distance of $90 \mathrm{~cm}$. As the data link requires a strict $\operatorname{LoS}$ operation, we introduced a visible-light localization technique to provide tracking information to the receiver. The angular resolution provided by the localization algorithm $\left(\Delta \Omega<1^{\circ}\right)$ allows to track the transmitter position within the angular tolerance of the LoS VLC link. The localization algorithm uses low frequency tones that do not overlap with the spectrum of the DMT data signal.

\section{REFERENCES}

[1] J. Llorca, A. Desai, U. Vishkin, C. Davis, and S. Milner, "Reconfigurable optical wireless sensor networks," in Proceedings of SPIE, vol. 5237, 2004, p. 136.

[2] K.-D. Langer, J. Grubor, O. Bouchet, M. El Tabach, J. Walewski, S. Randel, M. Franke, S. Nerreter, D. O'Brien, G. Faulkner, I. Neokosmidis, G. Ntogari, and M. Wolf, "Optical wireless communications for broadband access in home area networks," in Transparent Optical Networks, 2008. ICTON 2008. 10th Anniversary International Conference on, vol. 4, june 2008, pp. $149-154$.

[3] J. Vucic, C. Kottke, K. Habel, and K. Langer, "803 Mbit/s visible light WDM link based on DMT modulation of a single RGB LED luminary," in Optical Fiber Communication Conference. Optical Society of America, 2011.

[4] X. Liu, H. Makino, S. Kobayashi, and Y. Maeda, "Design of an indoor self-positioning system for the visually impaired-simulation with rfid and bluetooth in a visible light communication system," in Engineering in Medicine and Biology Society, 2007. EMBS 2007. 29th Annual International Conference of the IEEE. IEEE, 2007, pp. 1655-1658.

[5] X. Liu, H. Makino, and Y. Maeda, "Basic study on indoor location estimation using visible light communication platform," in Engineering in Medicine and Biology Society, 2008. EMBS 2008. 30th Annual International Conference of the IEEE. IEEE, 2008, pp. 2377-2380.

[6] J. Akella, M. Yuksel, and S. Kalyanaraman, "A relative ad hoc localization scheme using optical wireless," in Communication Systems Software and Middleware, 2007. COMSWARE 2007. 2nd International Conference on. IEEE, 2007, pp. 1-8.

[7] S. Hann, J. Kim, S. Jung, and C. Park, "White LED ceiling lights positioning systems for optical wireless indoor applications," in Optical Communication (ECOC), 2010 36th European Conference and Exhibition on. IEEE, pp. 1-3.

[8] K. Wang, A. Nirmalathas, C. Lim, and E. Skafidas, " $4 \times 12.5 \mathrm{~Gb} / \mathrm{s}$ wdm optical wireless communication system for indoor applications," Lightwave Technology, Journal of, vol. 29, no. 13, pp. 1988 -1996, july1, 2011.

[9] - "High-speed duplex optical wireless communication system for indoor personal area networks," Optics Express, vol. 18, no. 24, pp. 25 199-25 216, 2010.

[10] H. Liu, H. Darabi, P. Banerjee, and J. Liu, "Survey of wireless indoor positioning techniques and systems," Systems, Man, and Cybernetics, Part C: Applications and Reviews, IEEE Transactions on, vol. 37, no. 6, pp. 1067-1080, 2007. 
[11] H. Le Minh, D. O'Brien, G. Faulkner, L. Zeng, K. Lee, D. Jung, and Y. Oh, "80 Mbit/s visible light communications using pre-equalized white LED," in Optical Communication, 2008. ECOC 2008. 34th European Conference on. IEEE, 2008, pp. 1-2.

[12] D. O'Brien, L. Zeng, H. Le-Minh, G. Faulkner, J. Walewski, and S. Randel, "Visible light communications: Challenges and possibilities," in Personal, Indoor and Mobile Radio Communications, 2008. PIMRC 2008. IEEE 19th International Symposium on. IEEE, 2008, pp. 1-5.

[13] J. Grubor, S. Randel, K.-D. Langer, and J. Walewski, "Broadband information broadcasting using led-based interior lighting," Lightwave Technology, Journal of, vol. 26, no. 24, pp. 3883 -3892, dec.15, 2008.

[14] T. Komine and M. Nakagawa, "Fundamental analysis for visible-light communication system using led lights," Consumer Electronics, IEEE Transactions on, vol. 50, no. 1, pp. 100-107, 2004.

[15] T. Rappaport et al., Wireless communications: principles and practice. Prentice Hall PTR New Jersey, 1996, vol. 207.

[16] European Standard EN-12464-1: Lighting of indoor work places, 2003. 